\title{
Association of Serum Levels of Adipokines and Insulin With Risk of Barrett's Esophagus: A Systematic Review and Meta- Analysis
}

\author{
Apoorva Krishna Chandar ${ }^{\star}, \ddagger$, Swapna Devanna§, Chang Lu*, Siddharth Singh ${ }^{\S}$, Katarina \\ Greer $^{\star}, \ddagger$, Amitabh Chak ${ }^{\star}, \ddagger$, and Prasad G. lyer $§$ \\ "Division of Gastroenterology and Liver Diseases, Case Western Reserve University, Cleveland \\ ‡Digestive Health Institute, University Hospitals Case Medical Center, Cleveland, Ohio §Division of \\ Gastroenterology and Hepatology, Mayo Clinic, Rochester, Minnesota
}

\begin{abstract}
BACKGROUND \& AIMS-Metabolically active visceral fat may be associated with esophageal inflammation, metaplasia, and neoplasia. We performed a meta-analysis to evaluate the association of serum adipokines and insulin with Barrett's esophagus (BE).

METHODS-We performed a systematic search of multiple electronic databases, through April 2015, to identify all studies reporting associations between leptin, adiponectin, insulin, insulin resistance, and risk of $\mathrm{BE}$ in adults. Comparing the highest study-specific category with the reference category for each hormone, we estimated the summary adjusted odds ratio (aOR) and 95\% confidence intervals (CI), using a random effects model.
\end{abstract}

RESULTS-We identified 9 observational studies (10 independent cohorts; 1432 patients with BE total, and 3550 control subjects). Meta-analysis revealed that high serum level of leptin was associated with 2-fold higher risk of $\mathrm{BE}$ (BE cases vs population control subjects in 5 studies: aOR, 2.23; 95\% CI, 1.31-3.78; $\mathrm{I}^{2}, 59 \%$ ). Total serum level of adiponectin was not associated with BE (BE cases vs population control subjects in 5 studies: aOR, 0.79; 95\% CI, 0.46-1.34; $\mathrm{I}^{2}, 65 \%$ ), although 1 study observed decreased risk of BE with increased level of low-molecular-weight adiponectin. High serum level of insulin was associated with increased risk of BE (BE cases vs population control subjects in 3 studies: aOR, 1.74; 95\% CI, 1.14-2.65; $\mathrm{I}^{2}, 0$ ), whereas insulin resistance was not associated with increased risk of $\mathrm{BE}$ (BE cases vs gastroesophageal reflux disease control subjects in 2 studies: aOR, $0.98 ; 95 \%$ CI, 0.42-2.30; $\mathrm{I}^{2}, 64 \%$ ).

CONCLUSIONS-Increased serum levels of leptin and insulin are associated with increased risk of BE, compared with population control subjects. In contrast, increased total serum levels of

Reprint requests Address requests for reprints to: Prasad G. Iyer, MD, MS, Barrett's Esophagus Unit, Division of Gastroenterology and Hepatology, Mayo Clinic, 200 1st Street SW, Rochester, Minnesota 55905. iyer.prasad@ mayo.edu; fax: (507) 255-7612..

Supplementary Material

Note: To access the supplementary material accompanying this article, visit the online version of Clinical Gastroenterology and Hepatology at www.cghjournal.org, and at http://dx.doi.org/10.1016/j.cgh.2015.06.041.

Conflicts of interest

The authors disclose no conflicts. 
adiponectin and insulin do not seem to modify BE risk. Well-designed longitudinal studies of incident BE are needed to clarify existing associations of serum adipokines and insulin with BE.

\section{Keywords}

Barrett's Esophagus; Systematic Review; Meta-analysis; Adipokines; Leptin

The incidence of esophageal adenocarcinoma (EAC), a cancer with a dismal prognosis, continues to rise in the United States. ${ }^{1,2}$ Barrett's esophagus (BE), a premalignant condition characterized by change in the squamous mucosa of the esophagus to intestinalized columnar epithelium, is an established precursor of EAC. ${ }^{3}$ Obesity, and in particular central adiposity, has been identified as a risk factor for gastroesophageal reflux disease (GERD), $\mathrm{BE}$, and EAC. ${ }^{4-6}$ Obesity is thought to disrupt the gastroesophageal junction, promoting reflux induced inflammation that leads to $\mathrm{BE} .{ }^{7,8}$ However, obesity has also been implicated in the development of other cancers that do not have a mechanical explanation. ${ }^{9}$ Therefore, other independent molecular mechanisms and/or their interplay likely contribute to the increased risk of $\mathrm{BE}$ and its subsequent progression to cancer in obese persons.

Obesity represents a state of chronic low-level inflammation. Adipose tissue is metabolically active and produces adipocytokines or adipokines, such as adiponectin and leptin, which may play a role in regulating inflammation and cell proliferation. Although adiponectin is produced exclusively by adipose tissue, its levels are inversely correlated with abdominal obesity and body fat mass. ${ }^{10}$ Adiponectin levels have been found to be lower in men than women. ${ }^{11}$ It is known to have anti-inflamma-tory ${ }^{12}$ and antiangiogeneic ${ }^{13}$ properties and may influence tumorigenesis. Serum leptin concentration, however, is directly proportional to adipose tissue mass. ${ }^{14}$ Leptin levels are significantly higher in women than in men, even after accounting for total body fat mass. ${ }^{15}$ Leptin regulates neovascularization ${ }^{16}$ and also acts as an antiapopototic factor. ${ }^{17}$ Leptin has been shown to act synergistically with stomach acid and promotes proliferation and inhibits apoptosis in EAC cells. ${ }^{18}$ The relationship of adiponectin and leptin is complex with crosstalk between the 2 adipokines. Adiponectin may have an antagonistic effect on leptin by inhibiting leptin-induced cell proliferation. ${ }^{19}$

Insulin, a hormone secreted by the $\beta$ cells in the islets of Langerhans, has diverse functions including but not limited to cell metabolism, cellular degradation, and maintenance of vascular integrity. Serum insulin levels are particularly influenced by obesity, which leads to insulin resistance. ${ }^{20}$ Elevated insulin levels up-regulate the insulin/insulin-like growth factor signaling pathway, which inhibits apoptosis ${ }^{21}$ and promotes cell proliferation. Such an activation of the insulin-like growth factor pathway has been shown to occur in BE. ${ }^{22}$ Furthermore, both adiponectin and leptin are intimately connected with insulin resistance. ${ }^{23}$ Adiponectin increases peripheral tissue sensitivity to insulin and low adiponectin levels have been implicated in obesity-related insulin resistance. ${ }^{24}$ On the contrary, elevated serum leptin levels in the obese state have been shown to be associated with insulin resistance. ${ }^{25}$

Serum adipokines and insulin may modify the association between obesity and BE. Several observational studies have examined the association of serum adipokines and insulin with $\mathrm{BE}^{26-34}$ but evidence regarding this association remains inconclusive. Given the uncertainty around the overall body of evidence, we decided to conduct a systematic review and meta- 
analysis of observational studies evaluating the relationship between circulating levels of adiponectin, leptin, and insulin, and the risk of BE.

\section{Methods}

\section{Study Protocol}

This systematic review and meta-analysis was reported according to Preferred Reporting Items for Systematic Reviews and Meta-Analyses guidelines ${ }^{35}$ and conducted in accordance with the Cochrane Collaboration's systematic review framework. ${ }^{36}$ A comprehensive search of major electronic databases was conducted to identify all relevant studies on the association of circulating adipokines, leptin, and insulin levels and the risk of BE in adults from inception through April 2015. The following databases were searched: (1) PubMed, (2) Cumulative Index to Nursing and Allied Health Literature, (3) Excerpta Medica database, (4) Scopus, and (5) Cochrane Central Register of Controlled Trials (April 5, 2015). The search used the terms "adiponectin," "leptin," "insulin," "insulin resistance," "HOMA," "Barrett's esophagus," "case-control," "cross-sectional," "cohort," and "observational" in several combinations. The detailed search strategy for PubMed is presented in the Supplementary Appendix. In addition, abstracts presented at annual meetings of major gastroenterological societies (Digestive Diseases Week and American College of Gastroenterology) from 2010 to 2014 were hand-searched for eligibility.

Based on a priori defined study inclusion and exclusion criteria, 3 authors (AKC, SD, and SS) independently reviewed the titles and abstracts of studies identified in the search, to exclude studies that did not address the study question. Two reviewers (AKC and SS) performed data extraction in duplicate using a common data extraction template. Conflicts were resolved in consultation with 2 senior authors (AC and PGI). Inclusion was limited to case-control, cohort, and cross-sectional studies with a control group that evaluated the levels of adiponectin, leptin, insulin, and/or insulin resistance (estimated using the homeostatic model assessment for insulin resistance [HOMA-IR]) in patients with BE and a comparator population (population control subjects, screening colonos-copy control subjects, and/or GERD control subjects without endoscopic evidence of BE) and reported a measure of association (relative risk, odds ratio [OR]) along with a measure of variability (standard error, 95\% confidence interval [CI]). Population control subjects are subjects drawn from the community by using such methods as random digit dialing. It is estimated that $18 \%-28 \%$ of the general population has GERD symptoms. ${ }^{37}$ Screening colonoscopy control subjects are typically patients from the same institution where cases are selected, and are presumed to have a prevalence of GERD symptoms similar to population control subjects and hence are considered to be similar to population control subjects. GERD control subjects, however, have frequent and/or chronic GERD symptoms. They are often patients who are undergoing an endoscopy for GERD. Meta-analysis was restricted to studies that adjusted for age, sex, and a measure of obesity (body mass index [BMI], waistto-hip ratio [WHR], and/or waist circumference [WC]) given the interaction between these variables and the hormones under study.

The following data were extracted: 
1. Study characteristics: primary author, time period of study/year of publication, country, number of patients with BE and in the comparator group

2. Patient characteristics: age, sex, race/ethnicity, obesity (BMI, WHR, and/or WC), GERD duration and frequency, medication use (eg, nonsteroidal anti-inflammatory drugs and proton pump inhibitor), and smoking history

3. Type/length of BE (long segment/short segment) and type of BE dysplasia (lowgrade/high-grade dysplasia)

4. Mean values or range of values of serum adiponectin, leptin, insulin, and HOMAIR along with assays used for their measurement, presence of dose-response relationship

5. Study-specific point estimates (adjusted OR [aOR]) and 95\% CI or standard error for the association of serum adipokine, insulin and HOMA-IR with the risk of BE and potential confounding variables accounted for in the adjusted analyses

\section{Assessment of Study Quality}

The methodological quality of observational studies was independently assessed by 2 authors (AKC and SD), using a modified version of the Newcastle-Ottawa scale for observational studies. ${ }^{38}$ In this scale, studies were scored across 3 categories: selection (4 questions) and comparability of study groups ( 2 questions), and ascertainment of the exposure/outcome of interest ( 3 questions). This scale awards a maximum of 1 "star" for each item in the "selection" and "outcome" categories, and a maximum of 2 stars for "comparability." Accordingly, in the comparability category, if studies matched/controlled for age/gender, 1 star was awarded and if they also adjusted for obesity, an additional star was awarded. Cross-sectional studies were graded using the scale for case-control studies.

\section{Outcomes of Interest}

The primary outcome of interest was association between serum adiponectin (and its multimers), leptin, insulin, and insulin resistance and the risk of BE in comparison with population/screening colonoscopy control subjects. Secondary outcomes included examining the same associations using GERD control subjects and sex-specific associations of the different hormones with BE. Because GERD is recognized as an important risk factor for $\mathrm{BE}$ and could potentially confound any association between serum adipokines, insulin, and $\mathrm{BE}$, we performed meta-analysis of studies with a GERD control group. This also allowed us to assess if the association between BE and adipokines is mediated via or independent of GERD symptoms. Additionally, to examine the effect of gender on the association between serum adiopokines, insulin and BE, we also performed stratified meta-analysis of studies that reported such sexspecific associations.

\section{Statistical Analysis}

Using the generic inverse variance method, aORs from individual studies were pooled using a random effects model to assess the overall association of serum adiponectin, leptin, insulin, and HOMA-IR with risk of BE. Adjusted ORs for the highest category (ie, highest tertile or quartile) of adipokines and insulin values in comparison with the lowest category 
(ie, reference category) were summarized in this meta-analysis. When studies included more than 1 comparator, we conducted meta-analysis separately using the 2 comparators (ie, GERD and population control subjects). Screening colonoscopy control subjects were considered to be a reasonable approximation of population control subjects and we combined studies that used population control subjects and screening colonoscopy control subjects. When studies provided both matched and unmatched control groups, we decided a priori to use matched control groups. Studies that did not provide aORs were included in the qualitative analysis, but excluded from the quantitative analysis. Heterogeneity between studies was assessed by the $\mathrm{I}^{2}$ statistic as defined by the Cochrane handbook for systematic reviews. ${ }^{36}$ Accordingly, an $\mathrm{I}^{2}$ value of $50 \%$ or more was considered to represent substantial heterogeneity. Review manager 5 was used to generate forest plots, and funnel plots were used to test for potential publication bias. ${ }^{39}$ Sensitivity analysis was performed by excluding 1 study at a time to examine how that would influence the pooled effect estimate. Additional sensitivity analysis was performed based on the measure of obesity that was adjusted for in individual studies. We also conducted subgroup meta-analyses based on gender for BE risk association using studies that reported gender-specific aORs.

\section{Results}

The search strategy resulted in 667 citations with 9 articles (10 independent cohorts; 1432 BE patients and 3550 control patients [comprising 692 GERD control subjects and 2858 population control subjects]) eligible for inclusion. ${ }^{26-34}$ There were 10 cohorts in the 9 studies included in the analysis because 1 of the studies had a pilot and a validation cohort. Two studies ${ }^{40,41}$ were excluded from quantitative analysis because they reported only unadjusted values and another study ${ }^{42}$ was excluded because data were only available on gastric leptin levels. A flow diagram summarizing study identification and selection is shown in Supplementary Figure 1.

\section{Study Characteristics}

Study characteristics are described in Table 1 . The methodological quality of included studies is detailed in Table 2. All but 1 study were conducted in the United States, the 1 exception being an Australian study. ${ }^{30}$ One study was cross-sectional and the remaining 8 used a case-control study design; there were no longitudinal studies available on the association between level of serum adipokines and incident risk of developing BE. One study by Rubenstein et $\mathrm{al}^{31}$ used population control subjects, defined as either veterans or civilians without a diagnosis of BE, whereas another study by Rubenstein et $\mathrm{al}^{32}$ used GERD control subjects, defined as those reporting GERD symptoms and having a physician diagnosis of GERD. A third study by Rubenstein et $\mathrm{al}^{33}$ used a combination of GERD and non-GERD control subjects, defined as men with at least weekly GERD symptoms before use of antacids or men with at least Los Angeles Grade B esophagitis on upper endoscopy, and a random selection of colonoscopy screening control subjects without either GERD symptoms or endoscopic esophagitis. Garcia et al ${ }^{27}$ used screening colonoscopy control subjects who were scheduled for elective endoscopy; these control subjects were selected from primary care clinics at the same hospital where BE cases were selected. The 2 studies by Greer et $\mathrm{al}^{28,29}$ used 2 control groups: GERD control subjects were those with reflux 
symptoms without evidence of BE and a colonoscopy control group, which consisted of patients scheduled to undergo screening colonoscopy. Kendall et $\mathrm{al}^{30}$ used population control subjects who were randomly selected from the Australian electoral roll. Thompson et $\mathrm{al}^{34}$ also used population control subjects who were selected by random digit dialing. Almers et $\mathrm{al}^{26}$ used 2 control groups, a GERD control group that had an International Classification of Diseases-9 diagnosis of GERD and an upper endoscopy showing absence of columnar meta-plasia; the second population control group consisted of persons having a Kaiser Permanente membership and were randomly selected using risk set sampling. All included studies were at low risk of bias.

Overall, $65 \%$ of subjects were male (BE vs control subjects, $75 \%$ vs $61 \%$ ) and participants were mostly white (BE vs control subjects, $90 \%$ vs $77 \%$ ). All but 1 study included both incident and prevalent cases of BE; the study by Almers et $\mathrm{al}^{26}$ included only newly diagnosed BE cases. Average BMI was $29.6 \pm 7 \mathrm{~kg} / \mathrm{m}^{2}$ (BMI was reported as means and standard deviation in 6 studies). Sixty-three percent of the participants were either current or former smokers (BE vs control subjects, $67 \%$ vs $58 \%$ ).

\section{Association of Serum Adiponectin With Barrett's Esophagus}

Seven studies ${ }^{26-28,30-32,34}$ reported risk of BE in relation to total serum adiponectin. Metaanalysis showed that total serum adiponectin was not associated with risk of BE when compared with either population control subjects (5 studies; highest vs lowest category: aOR, $0.79 ; 95 \%$ CI, 0.46-1.34; $P=0.38$ ) or GERD control subjects (4 studies; highest vs lowest category: aOR, $1.20 ; 95 \% \mathrm{CI}, 0.69-2.10 ; P=0.52 ; \mathrm{I}^{2}, 60 \%$ ) (Figure 1). There was substantial heterogeneity between studies in both analyses $\left(\mathrm{I}^{2}>60 \%\right)$. Because of too few studies, funnel plot asymmetry could not be examined for publication bias.

Two studies ${ }^{26,32}$ reported association of high-molecular-weight (HMW) adiponectin with risk of BE. On meta-analysis, we observed that increased serum HMW adiponectin was associated with a $75 \%$ higher risk of BE when compared with GERD control subjects (highest vs lowest category: aOR, $1.75 ; 95 \% \mathrm{CI}, 1.16-2.63 ; P=0.008 ; \mathrm{I}^{2}, 0 \%$ ) (Supplementary Figure 2).

Three studies ${ }^{27,28,34}$ reported sex-specific associations of serum adiponectin with the risk of BE in comparison with population control subjects. Subgroup analysis revealed that the risk of $\mathrm{BE}$ in association with total serum adiponectin was not significantly different in males (highest vs lowest category: aOR, 0.71; 95\% CI, 0.28-1.78) or females (highest vs lowest category: aOR, $0.58 ; 95 \% \mathrm{CI}, 0.27-1.24)(P$ value for difference in subgroups $=0.74)$ (Supplementary Figure 3). Similarly, 3 studies ${ }^{26,28,32}$ reported sex-specific associations of serum adiponectin with the risk of BE in comparison with GERD control subjects. Subgroup analysis revealed that the risk of $\mathrm{BE}$ in association with total serum adiponectin was not significant when stratified by sex: males (highest vs lowest category: aOR, $0.91 ; 95 \% \mathrm{CI}$, 0.42-1.97) and females (highest vs lowest category: aOR, 1.71; 95\% CI, 0.78-3.72) ( $P$ value for difference in subgroups $=0.18)($ Supplementary Figure 4). 


\section{Association of Serum Leptin With Barrett's Esophagus}

Five studies (comprising 6 independent cohorts) 27,28,30,33,34 reported the association of serum leptin with BE risk. Meta-analysis of these 6 cohorts comparing BE cases with population control subjects showed that serum leptin was associated with a 2-fold increase in risk of $\mathrm{BE}$ (highest vs lowest category: aOR, $2.23 ; 95 \% \mathrm{CI}, 1.31-3.78 ; P=0.003$ ). There was substantial heterogeneity between studies $\left(\mathrm{I}^{2}, 59 \%\right)$. Only 1 study ${ }^{28}$ used GERD control subjects as a comparator and this study did not show a significant association between serum leptin and BE (OR, 1.32; 95\% CI, 0.61-2.87) (Figure 2). Availability of few studies meant that funnel plot asymmetry could not be examined to assess publication bias.

Four studies $27,28,30,34$ reported gender-specific aORs for the association of serum leptin with the risk of BE. Subgroup analysis comparing BE cases with population control subjects revealed there was no difference between males (highest vs lowest category; aOR, 2.02; 95\% CI, 0.85-4.81) and females (highest vs lowest category; aOR, 1.64; 95\% CI, 0.28-9.45) for the association between high serum leptin and BE risk ( $P$ value for difference in subgroups $=$ 0.83) (Supplementary Figure 5). Sex-specific associations comparing BE cases with GERD control subjects for the association between serum leptin and BE risk were only reported by Greer et $\mathrm{al}^{28}$ and as such, a subgroup analysis was not possible. In this study, the association between serum leptin and BE was not significant for either males (OR, 0.88; 95\% CI, 0.352.21) or females (OR, 4.42; 95\% CI, 0.71-27.46).

\section{Association of Serum Insulin With Barrett's Esophagus}

Four studies ${ }^{27,29,32,33}$ reported risk of BE in relation to serum insulin. Meta-analysis showed that serum insulin is associated with an increased risk of $\mathrm{BE}$ in comparison with population control subjects (highest vs lowest category: aOR, 1.74; 95\% CI, 1.14-2.65; $P=0.01$ ) (Figure 3$)$. There was no heterogeneity detected $\left(\mathrm{I}^{2}, 0 \%\right)$. Publication bias could not be assessed because of too few studies. When the analysis was limited to studies using GERD control subjects as comparator, ${ }^{29,32}$ we did not observe a significant association ( 2 studies; highest vs lowest category: aOR, 1.17; 95\% CI, 0.67-2.06; $\left.\mathrm{I}^{2}, 18 \%\right)$. Gender-stratified analysis was not possible because of limited data.

\section{Association of Insulin Resistance With Barrett's Esophagus}

Only 1 study using population control subjects provided associations of insulin resistance with BE. ${ }^{29}$ This study showed no association between insulin resistance and BE (highest vs lowest category; OR, 1.74; 95\% CI, 0.90-3.38). However, 2 studies $^{29,32}$ using GERD control subjects as comparator reported risk of BE with regards to HOMA-IR. Meta-analysis of these 2 studies also showed no significant association between insulin resistance and BE risk (highest vs lowest category: aOR, 0.98; 95\% CI, 0.42-2.30; $P=0.97$ ) (Figure 4). There was substantial heterogeneity between studies $\left(\mathrm{I}^{2}, 64 \%\right)$. Gender-stratified analysis was not possible because of paucity of available data.

\section{Sensitivity Analysis}

To investigate for possible sources of the heterogeneity, we conducted sensitivity analysis by excluding 1 study at a time. The study by Garcia et $\mathrm{a}^{27}$ strongly influenced the overall effect estimate in the meta-analysis of the association between serum leptin and BE risk. Although 
the association persisted after excluding this study, the summary estimate of effect was smaller with minimal heterogeneity (BE cases vs population control subjects; highest vs lowest category: aOR, 1.71; 95\% CI, 1.19-2.46; $P=0.001 ; \mathrm{I}^{2}, 15 \%$ ) (Supplementary Figure 6). Sensitivity analysis performed on the meta-analysis of other hormones (BE cases vs either population or GERD control subjects) showed that no 1 study in particular influenced the pooled effect estimate. We also performed additional sensitivity analysis based on the measure of obesity used in studies. All but 1 study adjusted for WHR or WC, the 1 exception being the study by Kendall et al, ${ }^{30}$ where BMI was used as an adjustment factor. Excluding this study (pilot and validation cohorts) from the adiponectin and leptin metaanalyses did not substantially affect the summary effect estimate in each.

\section{Effect of Gastroesophageal Reflux Disease}

Two studies ${ }^{26,33}$ provided GERD-stratified data (<weekly vs $\geq$ weekly GERD symptoms). In the study by Almers et $\mathrm{al}^{26}$ there were no significant associations between total serum adiponectin and BE risk in either of 2 strata. In the other study by Rubenstein et al ${ }^{33}$ persons who had weekly or more frequent GERD symptoms had an increased BE risk with elevated serum leptin (OR, 6.50; 95\% CI, 2.11-20) and insulin (OR, 2.59; 95\% CI, 1.09-6.12), whereas those with less than weekly symptoms showed no such association.

\section{Discussion}

This systematic review and meta-analysis demonstrates that increased leptin is associated with $\mathrm{BE}$ risk and may potentially play a role in the pathogenesis of $\mathrm{BE}$ in obese subjects. Risk of BE was twice as high in those with increased serum leptin levels. Similarly, we also found a significant association between increased serum insulin levels and BE risk. In this meta-analysis we did not find significant associations of BE with circulating levels of adiponectin and insulin resistance, however a higher risk of BE was seen with increased serum adiponectin HMW multimer.

Leptin has antiapoptotic ${ }^{17}$ and angiogenic ${ }^{16}$ effects. High serum leptin levels have been linked to the development and malignant progression of several cancers, such as breast, colon, prostrate, endometrium, and pancreas. ${ }^{43}$ Gastric leptin, however, has been found to maintain homeostasis of the gastric mucosa and regulate cellular proliferation, potentially preventing the formation of stomach ulcers. ${ }^{44}$ Francois et $\mathrm{al}^{42}$ found a significant association between increased gastric leptin levels and $\mathrm{BE}$ and also found the esophageal mucosa to be densely packed with leptin receptors despite lacking endogenous leptin production capability. Hence, they hypothesize that chronic gastric refluxate in conjunction with elevated gastric leptin is related to the cellular proliferation in BE. Our meta-analysis showed amplified positive association between serum leptin and BE only with population control subjects and not with GERD control subjects (although this was only with 1 study) suggesting that the association of BE with serum leptin may be mediated via increasing GERD. Additional studies are needed to assess if this association is independent of GERD. We did not find any evidence to suggest a gender-specific association.

Increased adiponectin levels did not influence BE risk, and there were no gender differences in risk association. The lack of association between total serum adiponectin and risk of $\mathrm{BE}$ 
could be related to the molecular weight of adiponectin measured in individual studies.

Three multimeric forms of adiponectin are recognized in circulating blood: low-molecularweight (LMW), middle-molecular-weight, and HMW adiponectin. ${ }^{45}$ The biologic effects of LMW and HMW multimers of adiponectin are distinct with anti-inflammatory and proinflammatory effects, respectively. Rubenstein et al ${ }^{32}$ found high levels of LMW adiponectin to be associated with a reduced risk of BE. This unique association could possibly be related to the inhibition of the proinflammatory cytokine interleukin- 6 and upregulation of anti-inflammatory cytokine interleukin-10 by LMW adiponectin. ${ }^{46}$ However, increased levels of HMW adiponectin were associated with increased BE risk in a recent study. ${ }^{26}$

Insulin and insulin-related signaling pathways have been shown to be up-regulated in $\mathrm{BE}$ and EAC tissue. ${ }^{22,47}$ Indeed insulin resistance (measured by HOMA-IR) is associated with progression to EAC in subjects with BE. ${ }^{48}$ Given that recent studies have shown association between $\mathrm{BE}$ and measures of central obesity ${ }^{5}$ and diabetes mellitus type $2,{ }^{49}$ it is conceivable that hyperinsulinemia and insulin resistance (which are known consequences of central obesity) are associated with BE pathogenesis. Our meta-analysis was able to partially corroborate these findings. Although we observed an increase in BE risk in association with serum insulin level when population control subjects were used, we did not find the same when GERD control subjects were used as the comparator. This may reflect the confounding effect of GERD on this association or the possibility of overmatching of GERD control subjects to BE cases. It is of note that in the study by Rubenstein et $\mathrm{a}^{33}$ the association of serum insulin with BE in nondiabetic male subjects (in comparison with GERD male control subjects) was attenuated by adjustment for serum leptin raising the possibility that the association between insulin and BE might be partially mediated by leptin. A genderstratified analysis could not be performed because of paucity of data: this may potentially be revealing given the strong male association of central obesity.

It is simplistic to assume that the effects of obesity on the development of BE are mediated by 1 single adipokine. Leptin and adiponectin seem to crosstalk and both of these adipokines also affect insulin-signaling pathways. Furthermore, obesity is a chronic inflammatory state associated with elevated levels of other cytokines, such as interleukin- 6 and tumor necrosis factor- $a .{ }^{50}$ Conceivably, any or all of these and other yet undiscovered molecular mediators may be involved in the development of BE and EAC. The association of leptin with BE as measured by this meta-analysis indicates that leptin might be an important contributor and support further studies on the effects of leptin on the leptin receptor in the proliferation of Barrett's epithelium.

This systematic review and meta-analysis has several strengths. First, we adhered to a rigorous systematic review methodology. Second, we assessed study quality/risk of bias using the Newcastle-Ottawa quality rating scale. Third, we investigated possible sources of heterogeneity by performing subgroup and sensitivity analyses. Fourth, we decided a priori that only studies that either matched or controlled for age/sex and obesity (BMI/WHR/WC) would be part of the meta-analysis, thereby further minimizing bias. Studies included in this systematic review and meta-analysis were of high quality. Participants were mostly overweight or obese, although all studies controlled for BMI, WHR, or WC. A large 
proportion of the population were smokers (>60\%) and smoking is a known risk factor for $\mathrm{BE},{ }^{51}$ but only 2 studies controlled for cigarette use in their analysis.

Our study also had several limitations. First, studies included in this systematic review and meta-analysis were all observational, and even after controlling for confounders and/or accurate matching, there is always some residual confounding present because of unmeasured confounders. Second, substantial heterogeneity was observed in our metaanalysis, which could be caused by differences in clinical characteristics (distribution of sex, obesity, GERD) and variability in measurement assays across studies. However, despite the high heterogeneity, the primary difference was in the magnitude of association and not the direction of association. Nevertheless, we investigated this heterogeneity by performing subgroup analysis based on gender and sensitivity analysis by excluding 1 study $^{27}$ that demonstrated a reduction of statistical heterogeneity. A third limitation was the lack of sufficient studies, particularly for associations of leptin and insulin when GERD control subjects were used and for insulin resistance, which prevented us from drawing firm conclusions about these associations. Finally, availability of few studies also meant that funnel plots could not be assessed for publication bias. Given the nascency of the field, reporting bias is possible, and as the field evolves, a better understanding will develop of the potential role of adipokines in development of $\mathrm{BE}$ and progression to EAC.

The goal of a meta-analysis is not only to make the estimates of effect more precise, but also to define areas that need further investigation. The results of this meta-analysis show that serum leptin and insulin are associated with $\mathrm{BE}$ but also suggest that the molecular mechanisms by which leptin might contribute to the development of BE need to be investigated. Leptin levels are known to be higher in women than men and the association does not explain why BE is much more common in men than women. This meta-analysis also found that studies to date are not adequate to assess gender-specific effects of adipokines or insulin on BE. Although total serum adiponectin and insulin resistance were not associated with BE, the meta-analysis indicates that there are insufficient studies to exclude an association. The association of obesity with BE is likely complex. Continued efforts that define the roles, if any, of these adipokines and insulin in the development of BE are required.

\section{Supplementary Material}

Refer to Web version on PubMed Central for supplementary material.

\section{Acknowledgments}

The authors thank Mr Larry J. Prokop, Medical Librarian at the Mayo Clinic Library, for helping in the literature search for this systematic review and meta-analysis. This work was presented as a poster at Digestive Diseases Week 2014, Chicago, Illinois.

Funding

Supported by the U54 CA163060 research grant from the National Cancer Institute. 


\section{Abbreviations used in this paper}

aOR

BE

BMI

CI

EAC

GERD

HMW

HOMA-IR

LMW

OR

WC

WHR adjusted odds ratio

Barrett's esophagus

body mass index

confidence interval

esophageal adenocarcinoma

gastroesophageal reflux disease

high molecular weight

homeostatic model assessment for insulin resistance

low molecular weight

odds ratio

waist circumference

waist-to-hip ratio

\section{References}

1. Siegel R, Ma J, Zou Z, et al. Cancer statistics, 2014. CA Cancer J Clin. 2014; 64:9-29. [PubMed: 24399786]

2. Thrift AP, Whiteman DC. The incidence of esophageal adenocarcinoma continues to rise: analysis of period and birth cohort effects on recent trends. Ann Oncol. 2012; 23:3155-3162. [PubMed: 22847812]

3. Shaheen N, Ransohoff DF. Gastroesophageal reflux, Barrett esophagus, and esophageal cancer: scientific review. JAMA. 2002; 287:1972-1981. [PubMed: 11960540]

4. Hampel H, Abraham NS, El-Serag HB. Meta-analysis: obesity and the risk for gastroesophageal reflux disease and its complications. Ann Intern Med. 2005; 143:199-211. [PubMed: 16061918]

5. Singh S, Sharma AN, Murad MH, et al. Central adiposity is associated with increased risk of esophageal inflammation, metaplasia, and adenocarcinoma: a systematic review and meta-analysis. Clin Gastroenterol Hepatol. 2013; 11:1399-1412. e7. [PubMed: 23707461]

6. Hoyo C, Cook MB, Kamangar F, et al. Body mass index in relation to oesophageal and oesophagogastric junction adenocarcinomas: a pooled analysis from the International BEACON Consortium. Int J Epidemiol. 2012; 41:1706-1718. [PubMed: 23148106]

7. Ayazi S, Hagen JA, Chan LS, et al. Obesity and gastroesophageal reflux: quantifying the association between body mass index, esophageal acid exposure, and lower esophageal sphincter status in a large series of patients with reflux symptoms. J Gastrointest Surg. 2009; 13:1440-1447. [PubMed: 19475461]

8. Pandolfino JE, El-Serag HB, Zhang Q, et al. Obesity: a challenge to esophagogastric junction integrity. Gastroenterology. 2006; 130:639-649. [PubMed: 16530504]

9. Calle EE, Rodriguez C, Walker-Thurmond K, et al. Overweight, obesity, and mortality from cancer in a prospectively studied cohort of U.S. adults. N Engl J Med. 2003; 348:1625-1638. [PubMed: 12711737]

10. Scherer PE. Adipose tissue: from lipid storage compartment to endocrine organ. Diabetes. 2006; 55:1537-1545. [PubMed: 16731815]

11. Coppola A, Marfella R, Coppola L, et al. Effect of weight loss on coronary circulation and adiponectin levels in obese women. Int J Cardiol. 2009; 134:414-416. [PubMed: 18378021] 
12. Wolf AM, Wolf D, Rumpold H, et al. Adiponectin induces the anti-inflammatory cytokines IL-10 and IL-1RA in human leukocytes. Biochem Biophys Res Commun. 2004; 323:630-635. [PubMed: 15369797]

13. Brakenhielm E, Veitonmaki N, Cao R, et al. Adiponectin-induced antiangiogenesis and antitumor activity involve caspase-mediated endothelial cell apoptosis. Proc Natl Acad Sci U S A. 2004; 101:2476-2481. [PubMed: 14983034]

14. Friedman JM, Halaas JL. Leptin and the regulation of body weight in mammals. Nature. 1998; 395:763-770. [PubMed: 9796811]

15. Havel PJ, Kasim-Karakas S, Dubuc GR, et al. Gender differences in plasma leptin concentrations. Nat Med. 1996; 2:949-950. [PubMed: 8782440]

16. Sierra-Honigmann MR, Nath AK, Murakami C, et al. Biological action of leptin as an angiogenic factor. Science. 1998; 281:1683-1686. [PubMed: 9733517]

17. Ogunwobi O, Mutungi G, Beales IL. Leptin stimulates proliferation and inhibits apoptosis in Barrett's esophageal adenocarcinoma cells by cyclooxygenase-2-dependent, prostaglandin-E2mediated transactivation of the epidermal growth factor receptor and c-Jun NH2-terminal kinase activation. Endocrinology. 2006; 147:4505-4516. [PubMed: 16740977]

18. Beales IL, Ogunwobi OO. Leptin synergistically enhances the anti-apoptotic and growthpromoting effects of acid in OE33 oesophageal adenocarcinoma cells in culture. Mol Cell Endocrinol. 2007; 274:60-68. [PubMed: 17618045]

19. Ogunwobi OO, Beales IL. Globular adiponectin, acting via adiponectin receptor-1, inhibits leptinstimulated oesophageal adenocarcinoma cell proliferation. Mol Cell Endocrinol. 2008; 285:43-50. [PubMed: 18313838]

20. Schwartz MW, Porte D Jr. Diabetes, obesity, and the brain. Science. 2005; 307:375-379. [PubMed: 15662002]

21. Kooijman R. Regulation of apoptosis by insulin-like growth factor (IGF)-I. Cytokine Growth Factor Rev. 2006; 17:305-323. [PubMed: 16621671]

22. Greer KB, Kresak A, Bednarchik B, et al. Insulin/insulin-like growth factor-1 pathway in Barrett's carcinogenesis. Clin Transl Gastroenterol. 2013; 4:e31. [PubMed: 23466464]

23. Silha JV, Krsek M, Skrha JV, et al. Plasma resistin, adiponectin and leptin levels in lean and obese subjects: correlations with insulin resistance. Eur J Endocrinol. 2003; 149:331-335. [PubMed: $14514348]$

24. Kadowaki T, Yamauchi T, Kubota N, et al. Adiponectin and adiponectin receptors in insulin resistance, diabetes, and the metabolic syndrome. J Clin Invest. 2006; 116:1784-1792. [PubMed: 16823476]

25. Yadav A, Kataria MA, Saini V, et al. Role of leptin and adiponectin in insulin resistance. Clin Chim Acta. 2013; 417:80-84. [PubMed: 23266767]

26. Almers LM, Graham JE, Havel PJ, et al. Adiponectin may modify the risk of Barrett's esophagus in patients with gastroesophageal reflux disease. Clin Gastroenterol Hepatol. 2015; 13:2256-2264. [PubMed: 25632808]

27. Garcia JM, Splenser AE, Kramer J, et al. Circulating inflamma-tory cytokines and adipokines are associated with increased risk of Barrett's esophagus: a case-control study. Clin Gastroenterol Hepatol. 2014; 12:229-238. e3. [PubMed: 23954649]

28. Greer KB, Falk GW, Bednarchik B, et al. Associations of serum adiponectin and leptin with Barrett's esophagus. Clin Gastroenterol Hepatol. 2015; 13:2265-2272. [PubMed: 25737442]

29. Greer KB, Thompson CL, Brenner L, et al. Association of insulin and insulin-like growth factors with Barrett's oesophagus. Gut. 2012; 61:665-672. [PubMed: 21930730]

30. Kendall BJ, Macdonald GA, Hayward NK, et al. Leptin and the risk of Barrett's oesophagus. Gut. 2008; 57:448-454. [PubMed: 18178609]

31. Rubenstein JH, Dahlkemper A, Kao JY, et al. A pilot study of the association of low plasma adiponectin and Barrett's esophagus. Am J Gastroenterol. 2008; 103:1358-1364. [PubMed: 18510610]

32. Rubenstein JH, Kao JY, Madanick RD, et al. Association of adiponectin multimers with Barrett's oesophagus. Gut. 2009; 58:1583-1589. [PubMed: 19570765] 
33. Rubenstein JH, Morgenstern H, McConell D, et al. Associations of diabetes mellitus, insulin, leptin, and ghrelin with gastroesophageal reflux and Barrett's esophagus. Gastroenterology. 2013; 145:1237-1244. e1-5. [PubMed: 23999171]

34. Thompson OM, Beresford SA, Kirk EA, et al. Serum leptin and adiponectin levels and risk of Barrett's esophagus and intestinal metaplasia of the gastroesophageal junction. Obesity (Silver Spring). 2010; 18:2204-2211. [PubMed: 20111023]

35. Moher D, Liberati A, Tetzlaff J, et al. Preferred reporting items for systematic reviews and metaanalyses: the PRISMA statement. BMJ. 2009; 339:b2535. [PubMed: 19622551]

36. The Cochrane Collaboration. Higgins, JPT.; Green, S., editors. Cochrane Handbook for Systematic Reviews of Interventions Version 5.1.0 [updated March 2011]. 2011. Available from www.cochrane-handbook.org

37. El-Serag HB, Sweet S, Winchester CC, et al. Update on the epidemiology of gastro-oesophageal reflux disease: a systematic review. Gut. 2014; 63:871-880. [PubMed: 23853213]

38. Wells, GA.; Shea, B.; O'Connell, D., et al. [2014 June 27] The Newcastle-Ottawa Scale (NOS) for assessing the quality if nonrandomized studies in meta-analyses. Available from: http:// www.ohri.ca/programs/clinical_epidemiology/oxford.htm

39. The Cochrane Collaboration. Review Manager (RevMan) [Computer program]. Version 5.3. The Nordic Cochrane Centre; Copenhagen: 2014.

40. Mokrowiecka A, Daniel P, Jasinska A, et al. Serum adiponectin, resistin, leptin concentration and central adiposity parameters in Barrett's esophagus patients with and without intestinal metaplasia in comparison to healthy controls and patients with GERD. Hepatogastroenterology. 2012; 59:2395-2399. [PubMed: 22944288]

41. Healy LA, Ryan AM, Pidgeon G, et al. Lack of differential pattern in central adiposity and metabolic syndrome in Barrett's esophagus and gastroesophageal reflux disease. Dis Esophagus. 2010; 23:386-391. [PubMed: 20353443]

42. Francois F, Roper J, Goodman AJ, et al. The association of gastric leptin with oesophageal inflammation and metaplasia. Gut. 2008; 57:16-24. [PubMed: 17761783]

43. Garofalo C, Surmacz E. Leptin and cancer. J Cell Physiol. 2006; 207:12-22. [PubMed: 16110483]

44. Konturek PC, Brzozowski T, Sulekova Z, et al. Role of leptin in ulcer healing. Eur J Pharmacol. 2001; 414:87-97. [PubMed: 11230999]

45. Suzuki S, Wilson-Kubalek EM, Wert D, et al. The oligomeric structure of high molecular weight adiponectin. FEBS Lett. 2007; 581:809-814. [PubMed: 17292892]

46. Schober F, Neumeier M, Weigert J, et al. Low molecular weight adiponectin negatively correlates with the waist circumference and monocytic IL-6 release. Biochem Biophys Res Commun. 2007; 361:968-973. [PubMed: 17678873]

47. Clemons NJ, Phillips WA, Lord RV. Signaling pathways in the molecular pathogenesis of adenocarcinomas of the esophagus and gastroesophageal junction. Cancer Biol Ther. 2013; 14:782-795. [PubMed: 23792587]

48. Duggan C, Onstad L, Hardikar S, et al. Association between markers of obesity and progression from Barrett's esophagus to esophageal adenocarcinoma. Clin Gastroenterol Hepatol. 2013; 11:934-943. [PubMed: 23466711]

49. Iyer PG, Borah BJ, Heien HC, et al. Association of Barrett's esophagus with type II diabetes mellitus: results from a large population-based case-control study. Clin Gastroenterol Hepatol. 2013; 11:1108-1114. e5. [PubMed: 23591277]

50. Kern PA, Ranganathan S, Li C, et al. Adipose tissue tumor necrosis factor and interleukin-6 expression in human obesity and insulin resistance. Am J Physiol Endocrinol Metab. 2001; 280:E745-751. [PubMed: 11287357]

51. Cook MB, Shaheen NJ, Anderson LA, et al. Cigarette smoking increases risk of Barrett's esophagus: an analysis of the Barrett's and Esophageal Adenocarcinoma Consortium. Gastroenterology. 2012; 142:744-753. [PubMed: 22245667] 


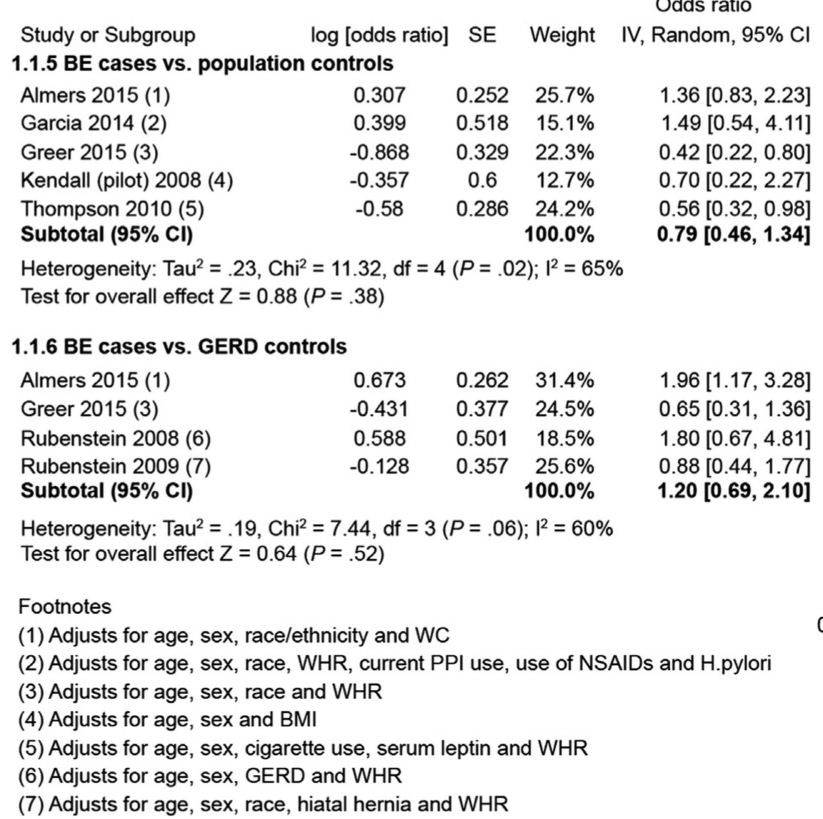

Figure 1.

Forest plot showing association between total serum adiponectin and BE risk in comparison with population control subjects (top) and with GERD control subjects (bottom). NSAIDs, nonsteroidal anti-inflammatory drugs; PPI, proton pump inhibitor; SE, standard error. 


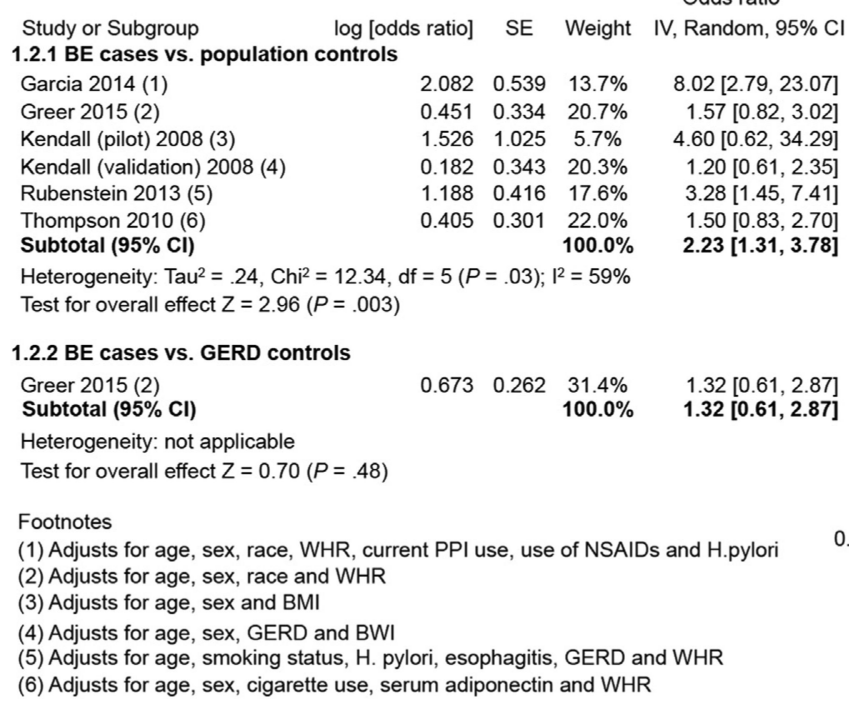

Figure 2.

Forest plot showing association between serum leptin and BE risk in comparison with population control subjects (top) and with GERD control subjects (bottom). NSAIDs, nonsteroidal anti-inflammatory drugs; PPI, proton pump inhibitor; SE, standard error. 


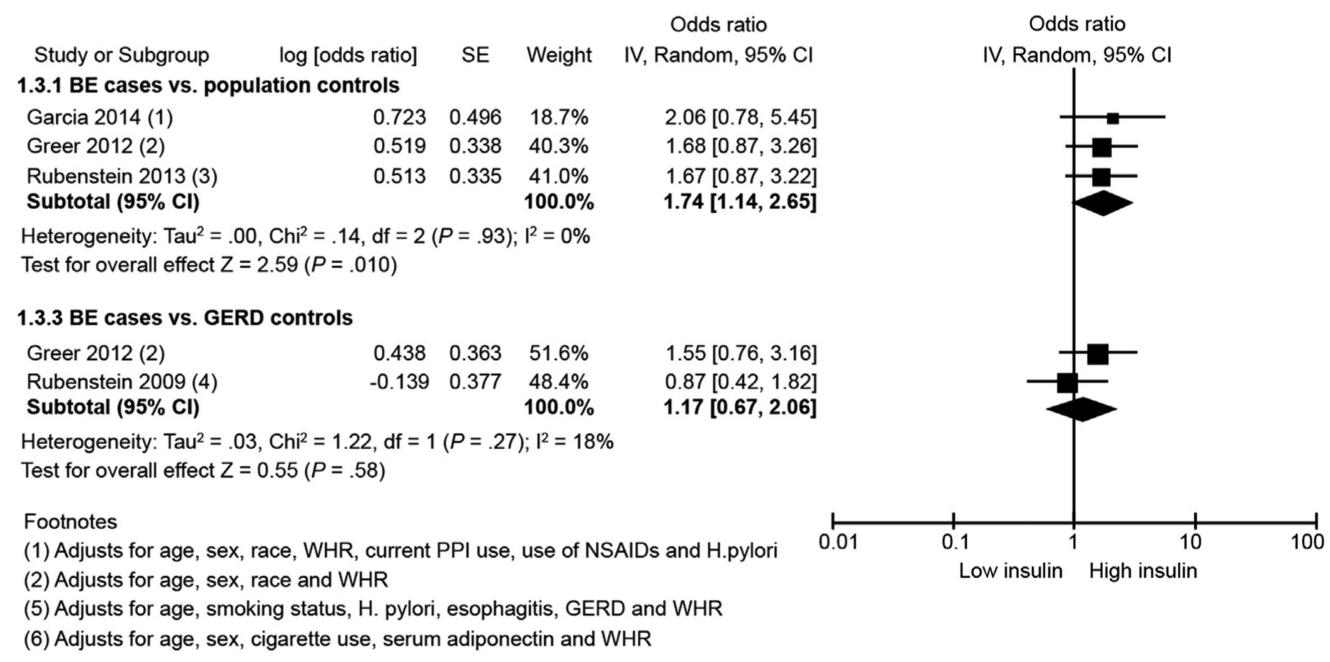

Figure 3.

Forest plot showing association between serum insulin and BE risk in comparison with population control subjects (top) and with GERD control subjects (bottom). NSAIDs, nonsteroidal anti-inflammatory drugs; PPI, proton pump inhibitor; SE, standard error. 


\begin{tabular}{|c|c|c|c|c|}
\hline \multirow{2}{*}{\multicolumn{2}{|c|}{$\begin{array}{l}\text { Study or Subgroup log [odds ratio] } \\
\text { 1.4.1 BE cases vs. population controls }\end{array}$}} & SE & Weight & $\begin{array}{l}\text { Odds ratio } \\
\text { IV, Random, } 95 \% \mathrm{Cl}\end{array}$ \\
\hline & & & & \\
\hline $\begin{array}{l}\text { Greer } 2012(1) \\
\text { Subtotal }(95 \% \mathrm{Cl})\end{array}$ & 0.554 & 0.338 & $\begin{array}{l}100.0 \% \\
100.0 \%\end{array}$ & $\begin{array}{l}1.74[0.90,3.38] \\
1.74[0.90,3.38]\end{array}$ \\
\hline \multicolumn{5}{|c|}{ Heterogeneity: not applicable } \\
\hline \multicolumn{5}{|c|}{ Test for overall effect $Z=1.64(P=.10)$} \\
\hline \multicolumn{5}{|c|}{ 1.4.4 BE cases vs. GERD controls } \\
\hline Greer $2012(1)$ & 0.405 & 0.356 & $51.3 \%$ & $1.32[0.61,2.87]$ \\
\hline Rubenstein 2009 (2) & -0.462 & 0.383 & $48.7 \%$ & $1.32[0.61,2.87]$ \\
\hline Subtotal $(95 \% \mathrm{Cl})$ & & & $100.0 \%$ & $1.32[0.61,2.87]$ \\
\hline
\end{tabular}

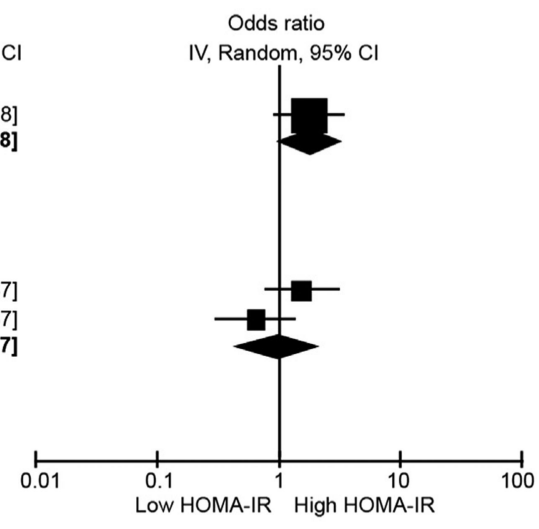

(1) Adjusts for age, sex, race and WHR

(1) Adjusts for age, sex, race and WHR
(2) Adjusts for age, sex, race, hiatal hernia and WHR

Figure 4.

Forest plot showing association between insulin resistance (HOMA-IR) and BE risk in comparison with population control subjects (top) and with GERD control subjects (bottom). SE, standard error. 


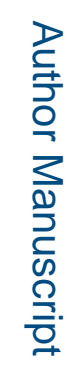

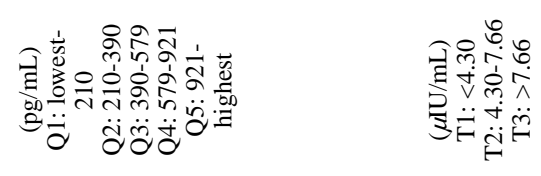

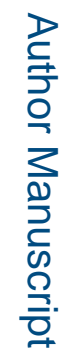

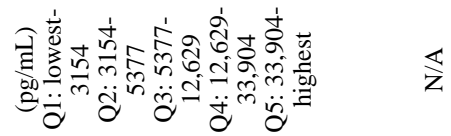

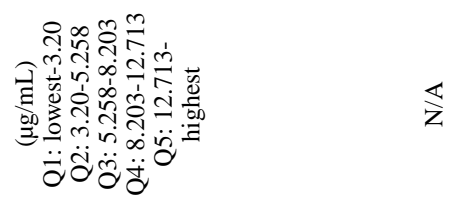

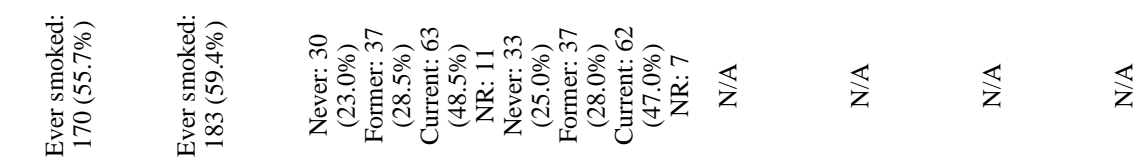

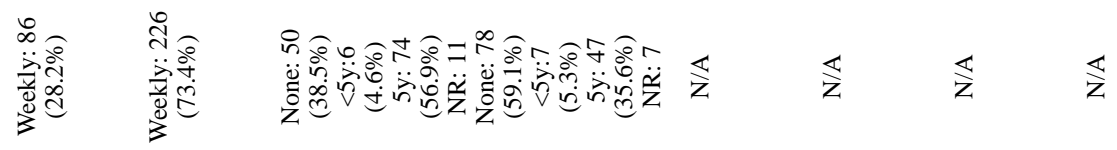

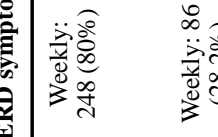

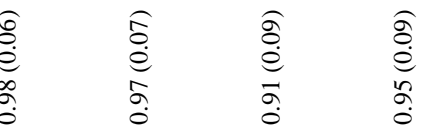

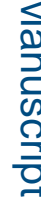

先

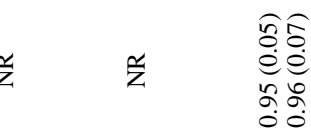

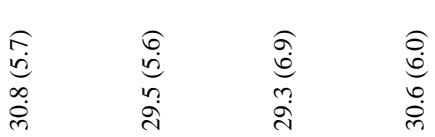

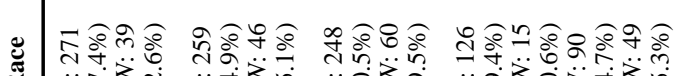

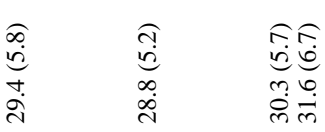

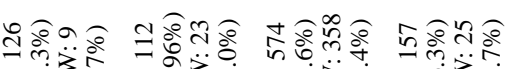

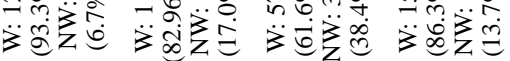

?

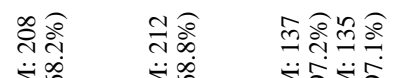

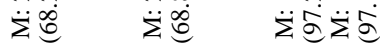

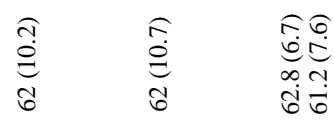

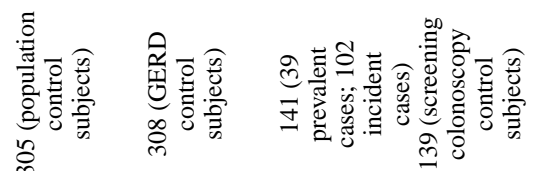

똟

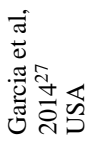

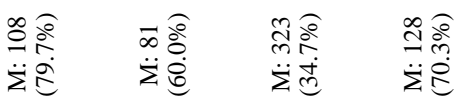

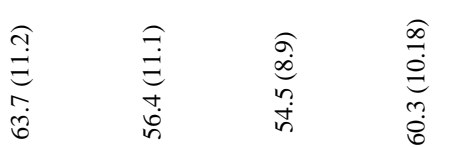

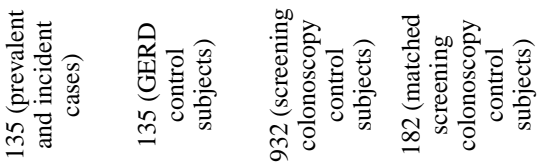

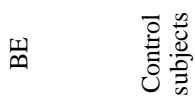

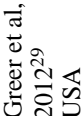




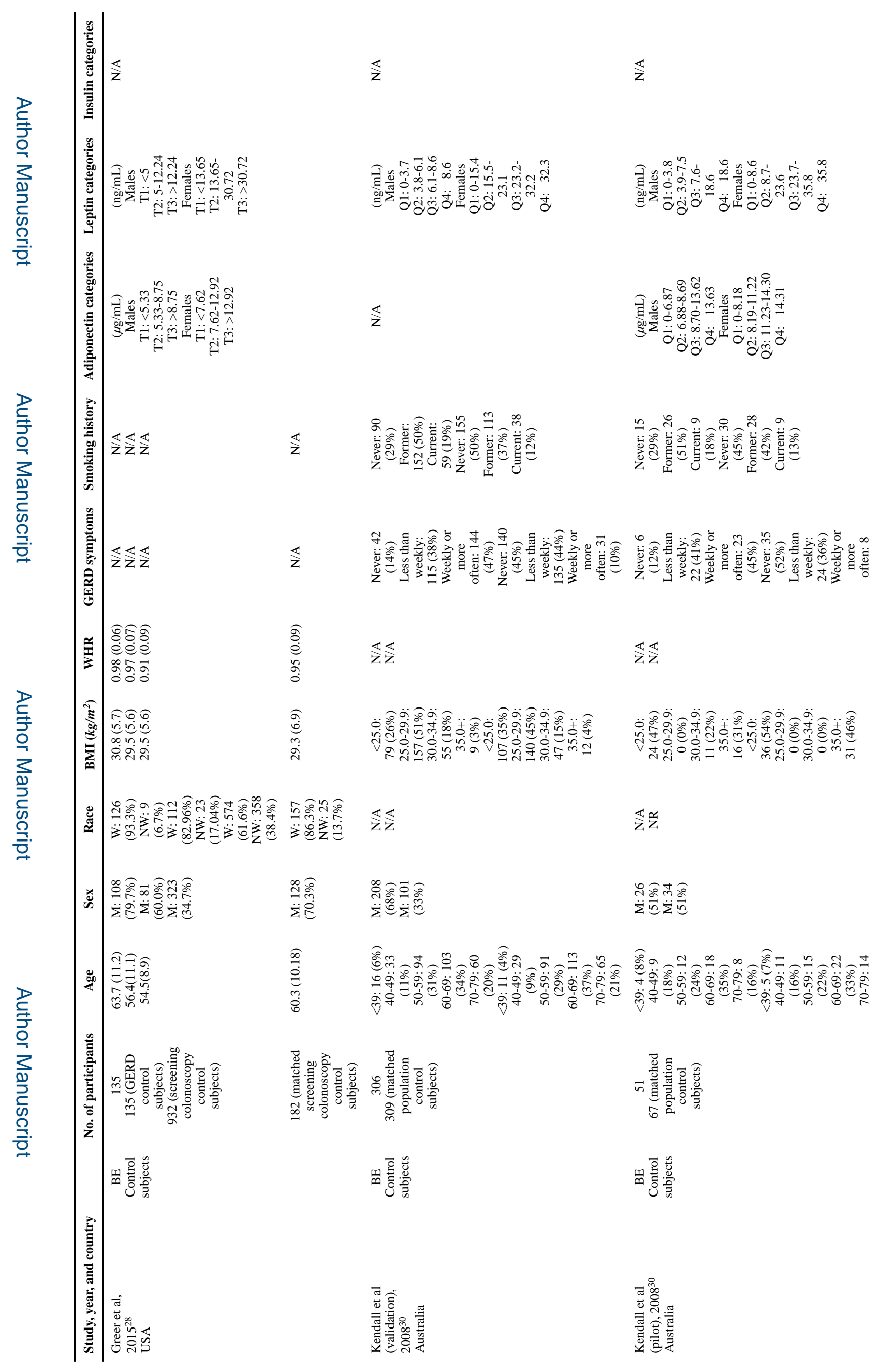




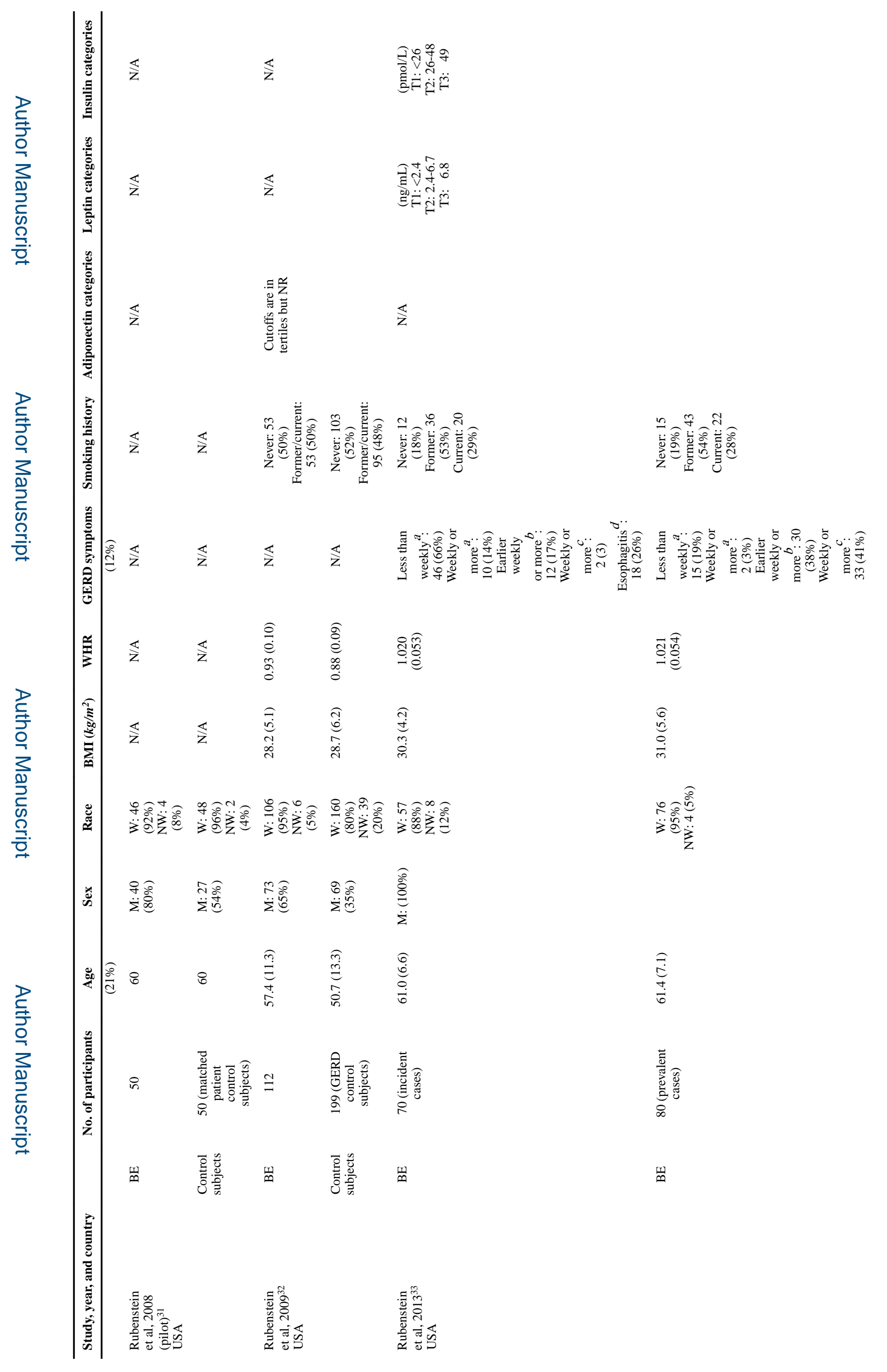




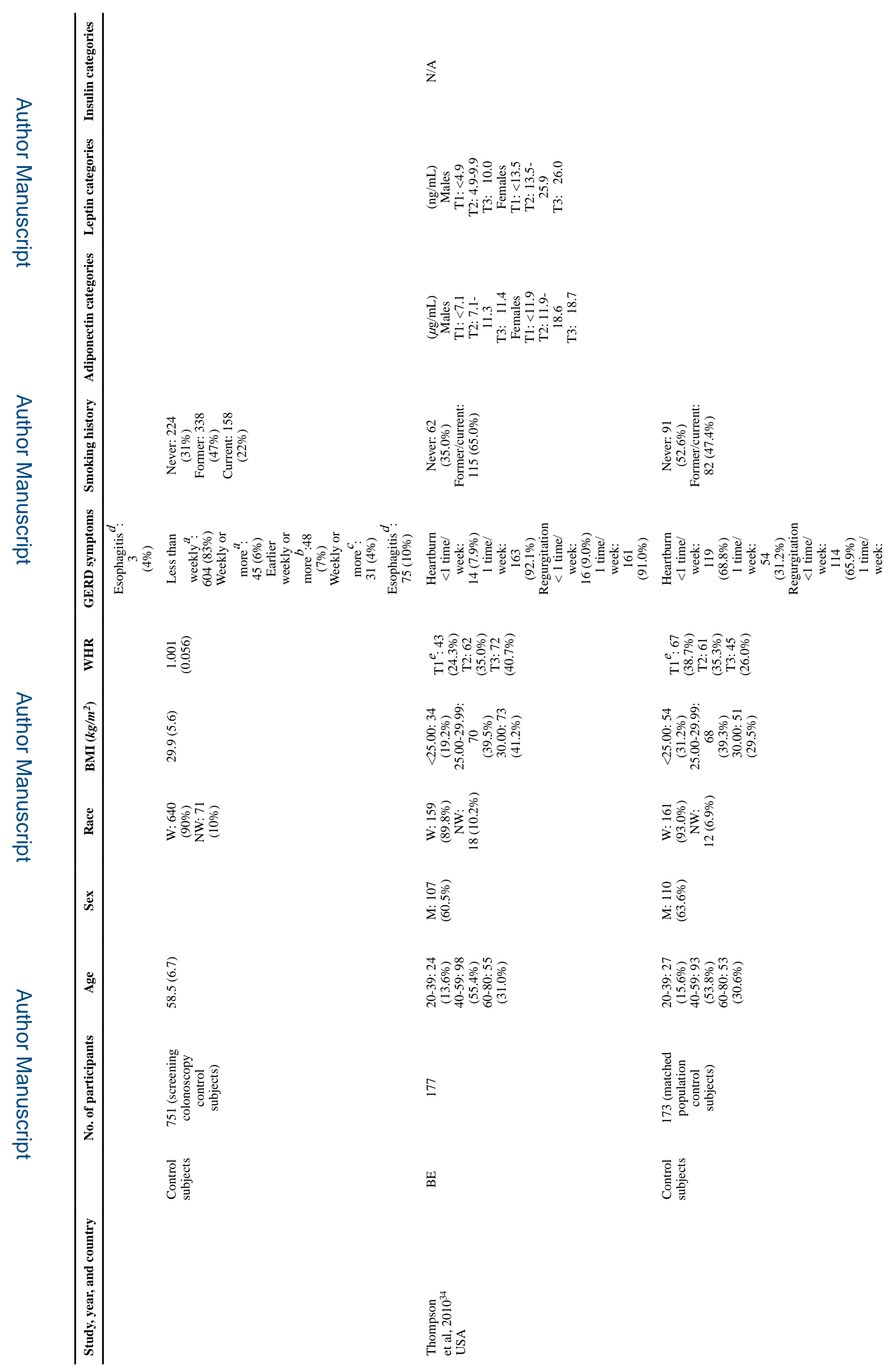




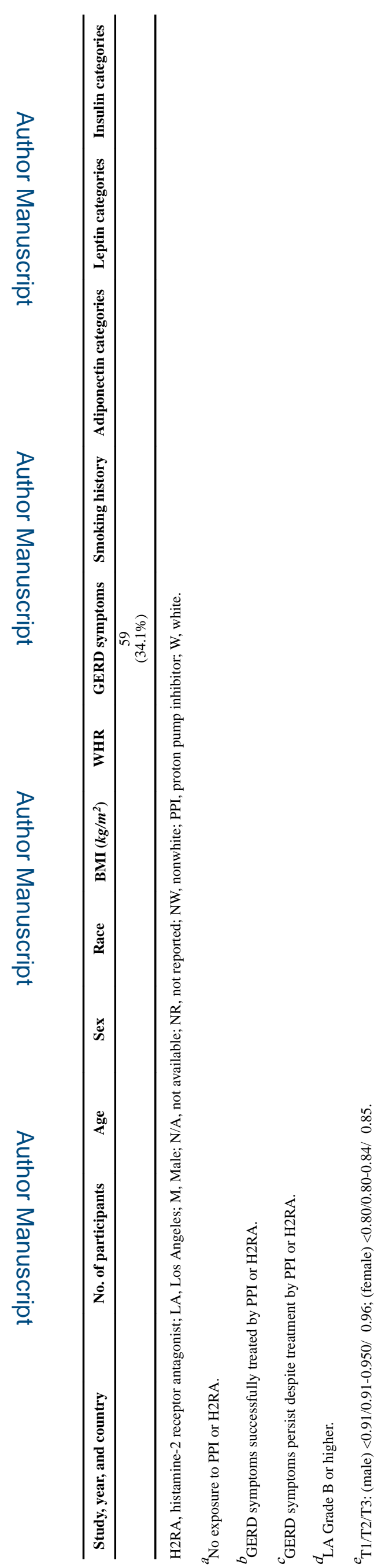




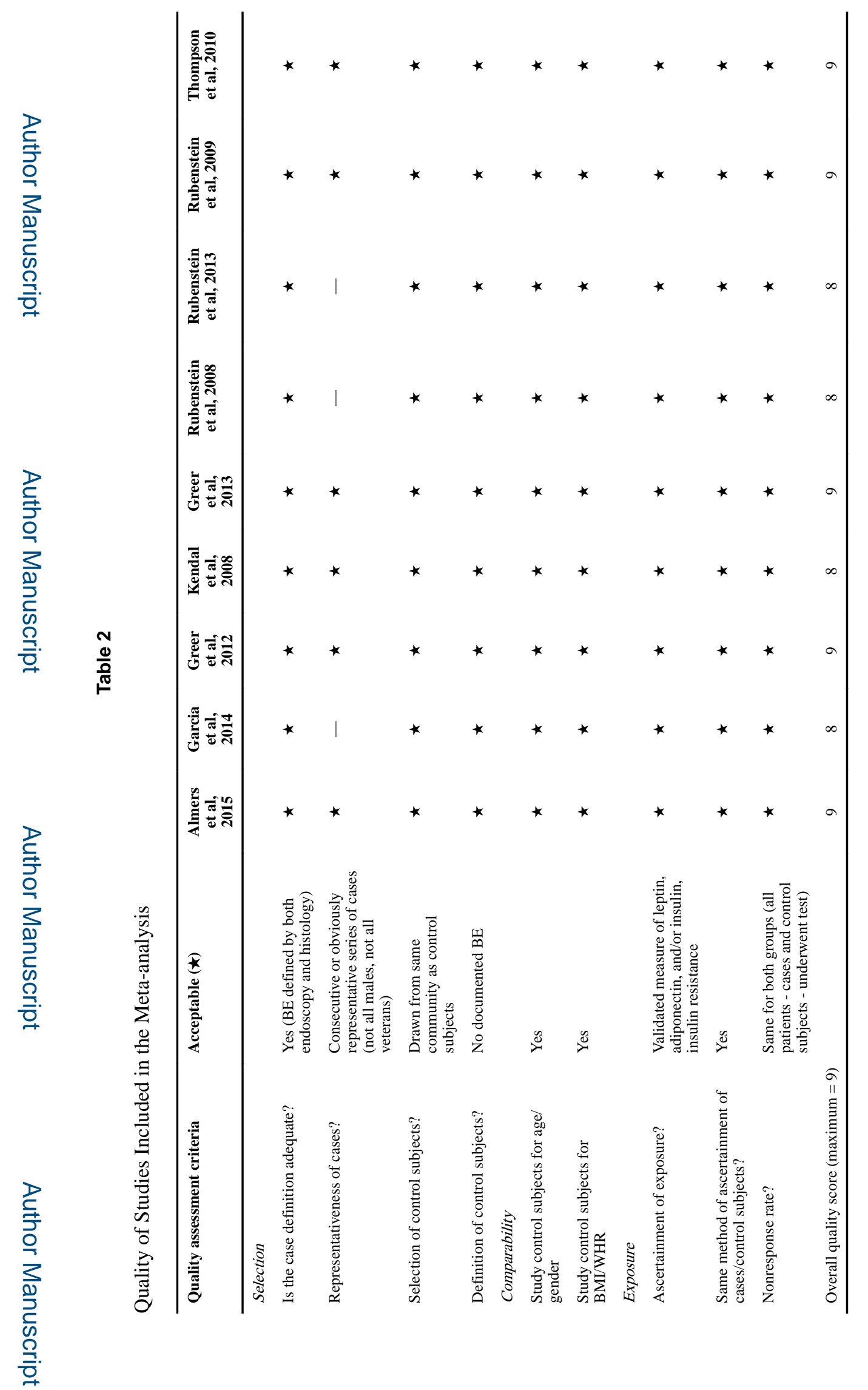

Clin Gastroenterol Hepatol. Author manuscript; available in PMC 2016 December 01. 